\title{
Systematic Transmission Electron Microscopy Study Investigating Lithium and Magnesium Intercalation in Vanadium Oxide Polymorphs
}

\author{
A. Mukherjee, ${ }^{1}$ R. F. Klie ${ }^{1}$, H.D. Yoo, ${ }^{2}$ G. Nolis, ${ }^{2}$ J. Cabana,${ }^{2}$ J. Andrews ${ }^{3}$ and S. Banerjee ${ }^{3}$ \\ 1. Department of Physics, University of Illinois at Chicago, 845 West Taylor Street, Chicago, IL \\ 2. Department of Chemistry, University of Illinois at Chicago, 845 West Taylor Street and Engineering \\ South (MC 111), Chicago, IL \\ 3. Department of Chemistry, Texas A\&M University, Ross Street, College Station, TX
}

Magnesium-ion based batteries promise a competitive alternative to conventional lithium-ion battery technology. Batteries combining Mg metal anode with a suitable intercalation-based cathode can offer much higher volumetric energy density, as well as significant cost and safety benefits over lithium ion batteries. Recent first-principles and experimental reports have established that orthorhombic $\alpha-\mathrm{V}_{2} \mathrm{O}_{5}$ is a promising intercalation cathode for $\mathrm{Mg}$ ion batteries. However, several crucial aspects of the intercalation phenomenon, such as the specific intercalation sites for $\mathrm{Mg}$ within $\alpha-\mathrm{V}_{2} \mathrm{O}_{5}$ or the formation of different phases upon $\mathrm{Mg}$ insertion into $\alpha-\mathrm{V}_{2} \mathrm{O}_{5}$ remain unclear. Further systematic characterization of the $\mathrm{Mg}$ intercalation behaviour is therefore required.

This contribution will focus on systematic investigation of $\mathrm{Mg}$ intercalation into $\alpha-\mathrm{V}_{2} \mathrm{O}_{5}$ by combining aberration-corrected scanning transmission electron microscopy (STEM) imaging, electron diffraction, electron energy loss (EEL) and energy dispersive x-ray spectroscopy (XEDS). More specifically, we will present a comparison of $\mathrm{Mg}$ insertion sites in two different samples: i) electrochemically cycled $\alpha-\mathrm{V}_{2} \mathrm{O}_{5}$ cathode in a prospective full cell vs $\mathrm{Mg}$ metal anode and ii) chemically synthesized $\mathrm{MgV}_{2} \mathrm{O}_{5}$ sample. In the case of electrochemically cycled $\alpha-\mathrm{V}_{2} \mathrm{O}_{5}$, our results determine the $\mathrm{Mg}$ intercalation sites and it is concluded that this sample exhibits the local formation of the $\varepsilon-\mathrm{Mg}_{0.5} \mathrm{~V}_{2} \mathrm{O}_{5}$ phase, as predicted by earlier first-principles density functional theory (DFT) calculations [1]. Figure 1a) and b) present atomic resolution high-angle annular dark-field (HAADF) and annular bright-field (ABF) images, respectively, for the electrochemically-cycled orthorhombic $\alpha-\mathrm{V}_{2} \mathrm{O}_{5}$ cathode. Simulated HAADF and ABF images for the DFT predicted $\varepsilon-\mathrm{Mg}_{0.5} \mathrm{~V}_{2} \mathrm{O}_{5}$ phase are overlaid on the experimental STEM images. The structural model for the $\varepsilon-\mathrm{Mg}_{0.5} \mathrm{~V}_{2} \mathrm{O}_{5}$ phase is shown in Fig 1(c). We will also show that the chemically synthesized sample presents the $\delta-\mathrm{MgV}_{2} \mathrm{O}_{5}$ phase [2].

Recent theoretical calculations have also predicted that the migration barrier for ionic intercalation can be decreased by exploiting different anion coordination environments in metastable vanadium oxide polymorphs, such as $\zeta-\mathrm{V}_{2} \mathrm{O}_{5}$ [3]. Figure 2a) presents atomic-resolution HAADF image for $\zeta_{-}-\mathrm{V}_{2} \mathrm{O}_{5}$ nanowires clearly showing the heavier $\mathrm{V}$ atoms and elucidating the tunnel structure for this novel polymorph; a structural model for the $\zeta-\mathrm{V}_{2} \mathrm{O}_{5}$ phase is presented in Figure $2 \mathrm{~b}$ ). We have investigated the lithium intercalation in this tunnel-structured $\zeta-\mathrm{V}_{2} \mathrm{O}_{5}$ polymorph, and will focus on showing that $\zeta-\mathrm{V}_{2} \mathrm{O}_{5}$ nanowires show much better Li-cycling properties (i.e. reversibility) compared to orthorhombic $\alpha-\mathrm{V}_{2} \mathrm{O}_{5}$ [4]. Moreover, $\mathrm{Mg}$ intercalation into $\zeta-\mathrm{V}_{2} \mathrm{O}_{5}$ nanowires will be investigated in detail, comparing electrochemical performance at both low and high temperature cycling followed by systematic STEM characterization. The results obtained for this novel polymorph $\zeta_{5}-\mathrm{V}_{2} \mathrm{O}_{5}$ will be directly compared with our previous work investigating $\mathrm{Mg}$ intercalation in $\alpha-\mathrm{V}_{2} \mathrm{O}_{5}$ [2]. Other $\mathrm{V}_{2} \mathrm{O}_{5}$ polymorphs, such as $\varepsilon-\mathrm{V}_{2} \mathrm{O}_{5}$ will also be tested for their ability to intercalate $\mathrm{Li}$ or $\mathrm{Mg}$ [5]. 
References:

[1] G. S. Gautam et al, Chemistry of Materials 10, (2015), p. 1021.

[2] A. Mukherjee et al, Chemistry of Materials 2017 (in print)

[3] A. Parija et al, Chemistry of Materials 28 (2016), p. 5611.

[4] A. Mukherjee et al, Direct observation of reversible topotactic lithium intercalation in tunnel structure $\zeta-\mathrm{V}_{2} \mathrm{O}_{5}$ nanowire cathode, (in preparation)

[5] This work is supported by the Joint Center for Energy Storage Research (JCESR), an Energy Innovation Hub funded by the U.S. Department of Energy (DOE), Office of Science, Basic Energy Sciences.

(a)

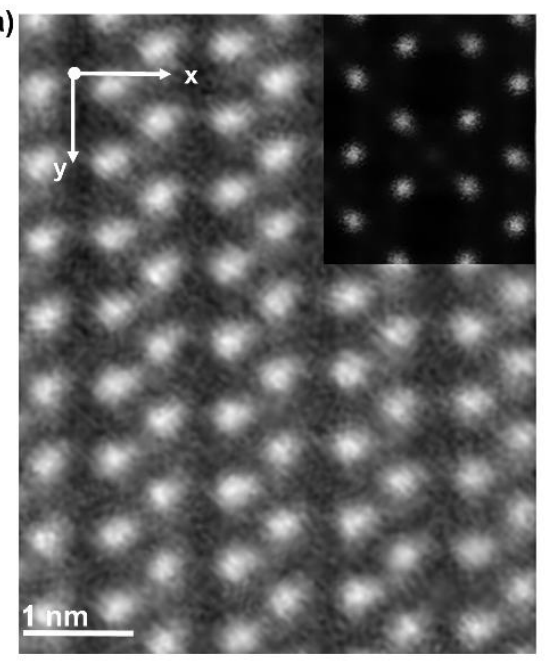

(b)

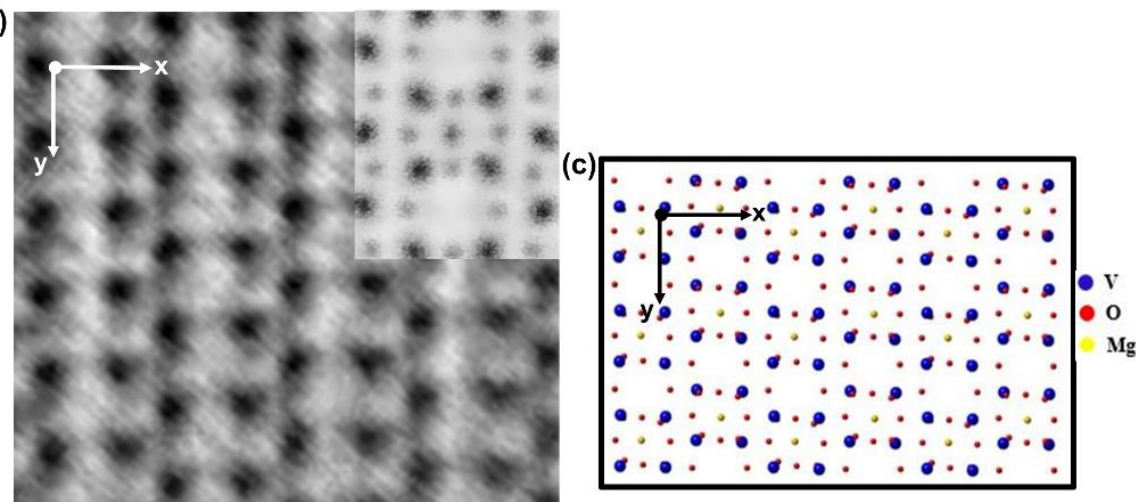

Figure 1. (a) Filtered atomic-resolution HAADF image of electrochemically cycled $\alpha-\mathrm{V}_{2} \mathrm{O}_{5}$ cathode, simulated image overlaid (b) Filtered ABF image, simulated image overlaid (c) Structural model for DFT predicted $\varepsilon-\mathrm{Mg}_{0.5} \mathrm{~V}_{2} \mathrm{O}_{5}$ phase in [001] zone

(a)
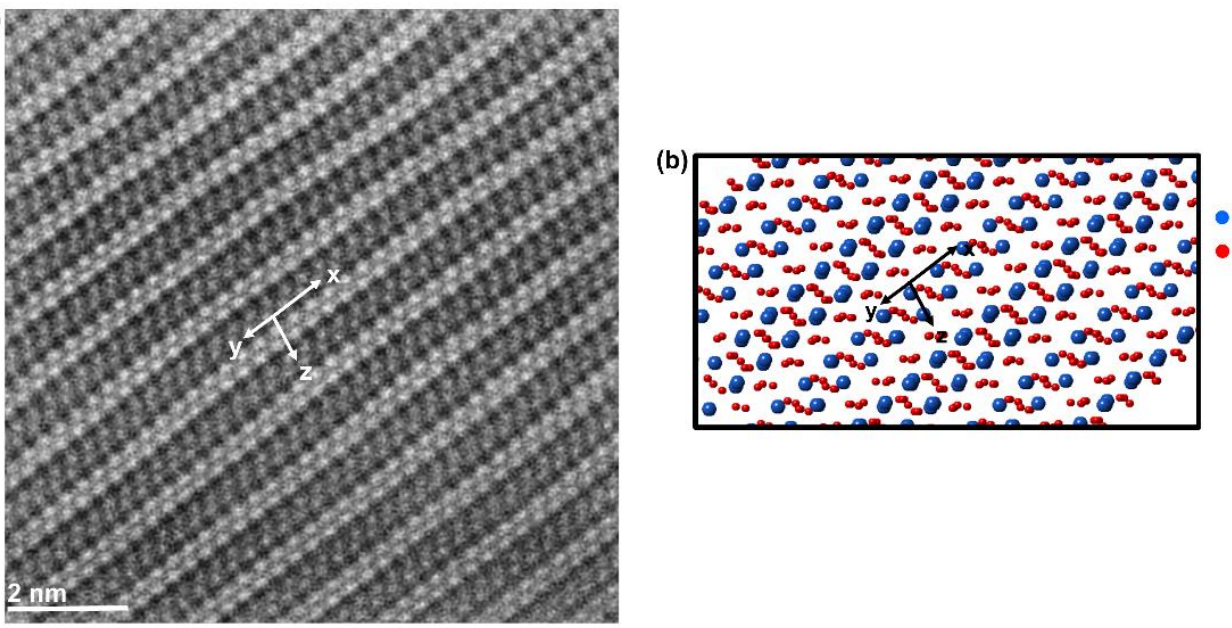

Figure 2. (a) Filtered atomic-resolution

HAADF image for pristine $\quad \zeta-\mathrm{V}_{2} \mathrm{O}_{5}$ nanowire (b) Structural model for $\zeta-\mathrm{V}_{2} \mathrm{O}_{5}$ in $\overline{[1} \overline{5} 0]$ orientation 